\title{
Application of artificial intelligence in anterior segment ophthalmic diseases: diversity and standardization
}

\author{
Xiaohang Wu ${ }^{1 \#}$, Lixue Liu ${ }^{1 \#}$, Lanqin Zhao ${ }^{1}$, Chong Guo ${ }^{1}$, Ruiyang Li ${ }^{1}$, Ting Wang ${ }^{1}$, Xiaonan Yang ${ }^{1}$, \\ Peichen Xie ${ }^{2}$, Yizhi Liu ${ }^{1}$, Haotian Lin ${ }^{1,3}$ \\ ${ }^{1}$ State Key Laboratory of Ophthalmology, Zhongshan Ophthalmic Center, ${ }^{2}$ Zhongshan School of Medicine, ${ }^{3}$ Center for Precision Medicine, Sun \\ Yat-sen University, Guangzhou, China \\ Contributions: (I) Conception and design: H Lin, X Wu; (II) Administrative support: None; (III) Provision of study materials or patients: None; (IV) \\ Collection and assembly of data: None; (V) Data analysis and interpretation: None; (VI) Manuscript writing: All authors; (VII) Final approval of \\ manuscript: All authors. \\ "These authors contributed equally to this work. \\ Correspondence to: Haotian Lin, Yizhi Liu. Zhongshan Ophthalmic Center, Sun Yat-sen University, 54\# Xianlie Road, Guangzhou, China. \\ Email: haot.lin@hotmail.com; yizhi_liu@aliyun.com.
}

\begin{abstract}
Artificial intelligence (AI) based on machine learning (ML) and deep learning (DL) techniques has gained tremendous global interest in this era. Recent studies have demonstrated the potential of AI systems to provide improved capability in various tasks, especially in image recognition field. As an imagecentric subspecialty, ophthalmology has become one of the frontiers of AI research. Trained on optical coherence tomography, slit-lamp images and even ordinary eye images, AI can achieve robust performance in the detection of glaucoma, corneal arcus and cataracts. Moreover, AI models based on other forms of data also performed satisfactorily. Nevertheless, several challenges with AI application in ophthalmology have also arisen, including standardization of data sets, validation and applicability of AI models, and ethical issues. In this review, we provided a summary of the state-of-the-art AI application in anterior segment ophthalmic diseases, potential challenges in clinical implementation and our prospects.
\end{abstract}

Keywords: Artificial intelligence (AI); anterior eye segment; computer-assisted diagnosis; machine learning (ML)

Submitted Jan 21, 2020. Accepted for publication Apr 17, 2020.

doi: 10.21037/atm-20-976

View this article at: http://dx.doi.org/10.21037/atm-20-976

\section{Introduction}

First conceptualized in 1956 by Dartmouth scholar John McCarthy, artificial intelligence (AI) is a general term referring to hardware or software that exhibits intelligent behavior (1). As a subset of AI, machine learning (ML) was proposed subsequently in 1959 and stated that "the computer should have the ability to learn using various statistical techniques, without being explicitly programmed" (2). ML has been widely adopted in applications such as computer vision and predictive analytics using complex mathematical models. Deep learning (DL) is a class of state-of-the-art machine learning techniques that has sparked tremendous global interest in recent years (3).
With improved performance, the deployment of DL focuses on image recognition, speech recognition and natural language processing $(4,5)$.

Healthcare has become one of the frontiers of AI application in recent years, particularly for those imagecentric subspecialties such as radiology, dermatology, pathology and ophthalmology (6). For example, AI algorithms have proved effective in the detection of pulmonary tuberculosis from chest radiographs and melanoma on digital skin photographs $(7,8)$. Similarly, in ophthalmology, AI has been primarily applied to image-based diagnosis of diabetic retinopathy, glaucoma, age-related macular degeneration and retinopathy of 


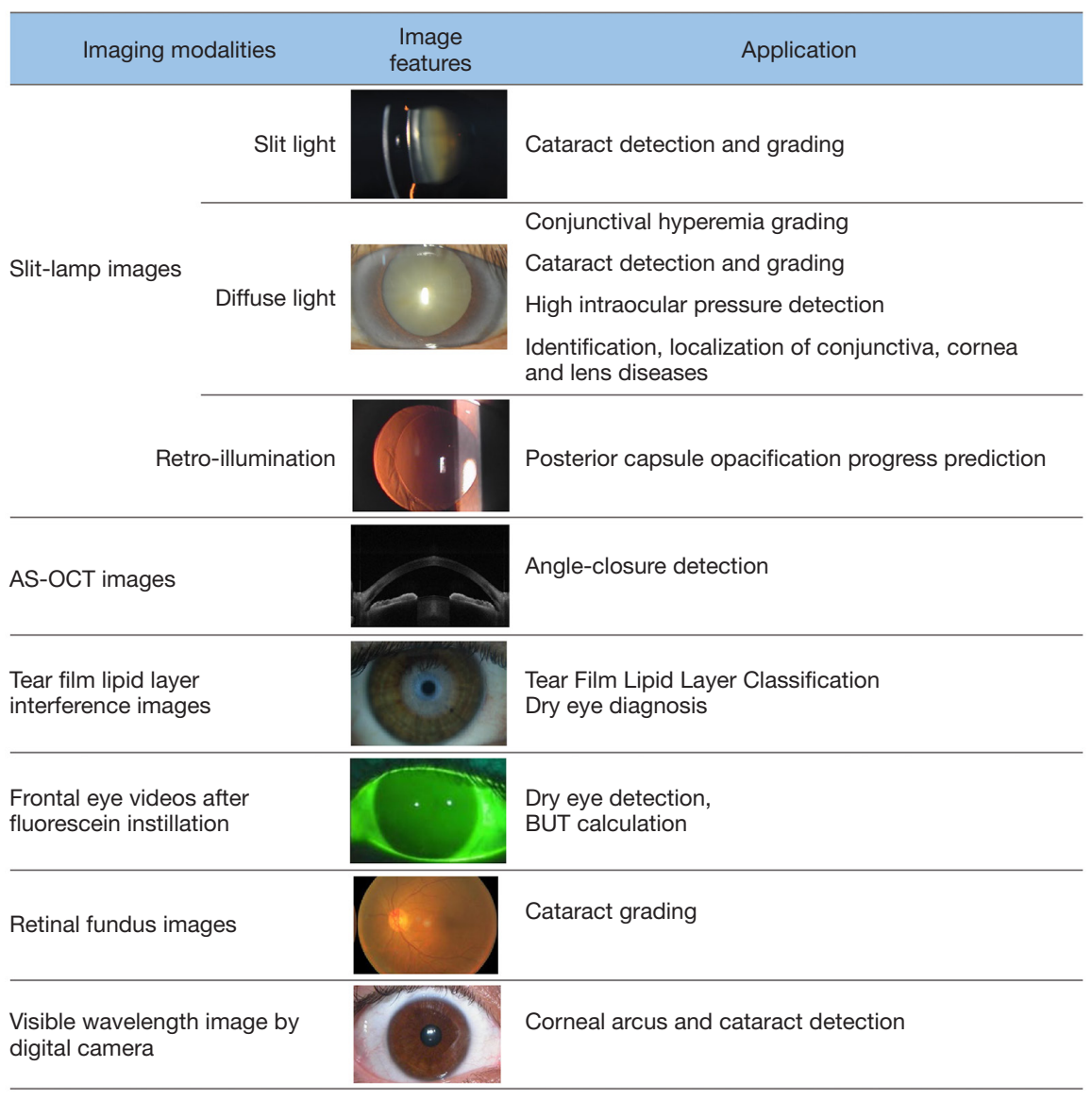

Figure 1 The ophthalmic imaging modalities in $\mathrm{AI}$ applications in anterior segment ocular diseases.

prematurity, most of which are retinal diseases (9).

Unlike the diagnosis of retinal diseases, which is largely dependent on ophthalmoscopy, multiple examinations are required to diagnose anterior segment diseases given the complexity of its structure and physiological functions. Anterior eye segment is defined as the front third of the eye that includes conjunctiva, cornea, anterior chamber, iris, pupil, ciliary body, and lens. These structures constitute the path of light through the eye and the ocular refractive system. To establish a definite diagnosis of anterior segment eye diseases, evaluation of both anatomy and function are necessitated, including slit-lamp biomicroscopy, optical coherence tomography, corneal topography, tonometry, perimetry, etc. Accordingly, various forms of data besides images, such as videos, formatted parameters and texts, have been used in the AI-assisted detection of these diseases. This review summarizes the application of AI systems in anterior segment ophthalmic diseases, potential challenges in clinical adoption and possible paths forward.

\section{Constructing models}

Multiple ophthalmic imaging modalities, such as slit-lamp images (10-17), AS-OCT images (18-23) and tear film lipid layer interference images (24-27), have been widely used in AI for the diagnosis of anterior segment ocular diseases. In addition, structured data, such as corneal parameters (28-33), have been used as well. The ophthalmic imaging modalities used in diagnosis are illustrated in Figure 1.

The steps for building an AI model include the following phases: data preparation, data partition, model optimization and evaluation of the system, as showed in Figure 2.

\section{Data preparation}

In order to improve the performance of models or meet 


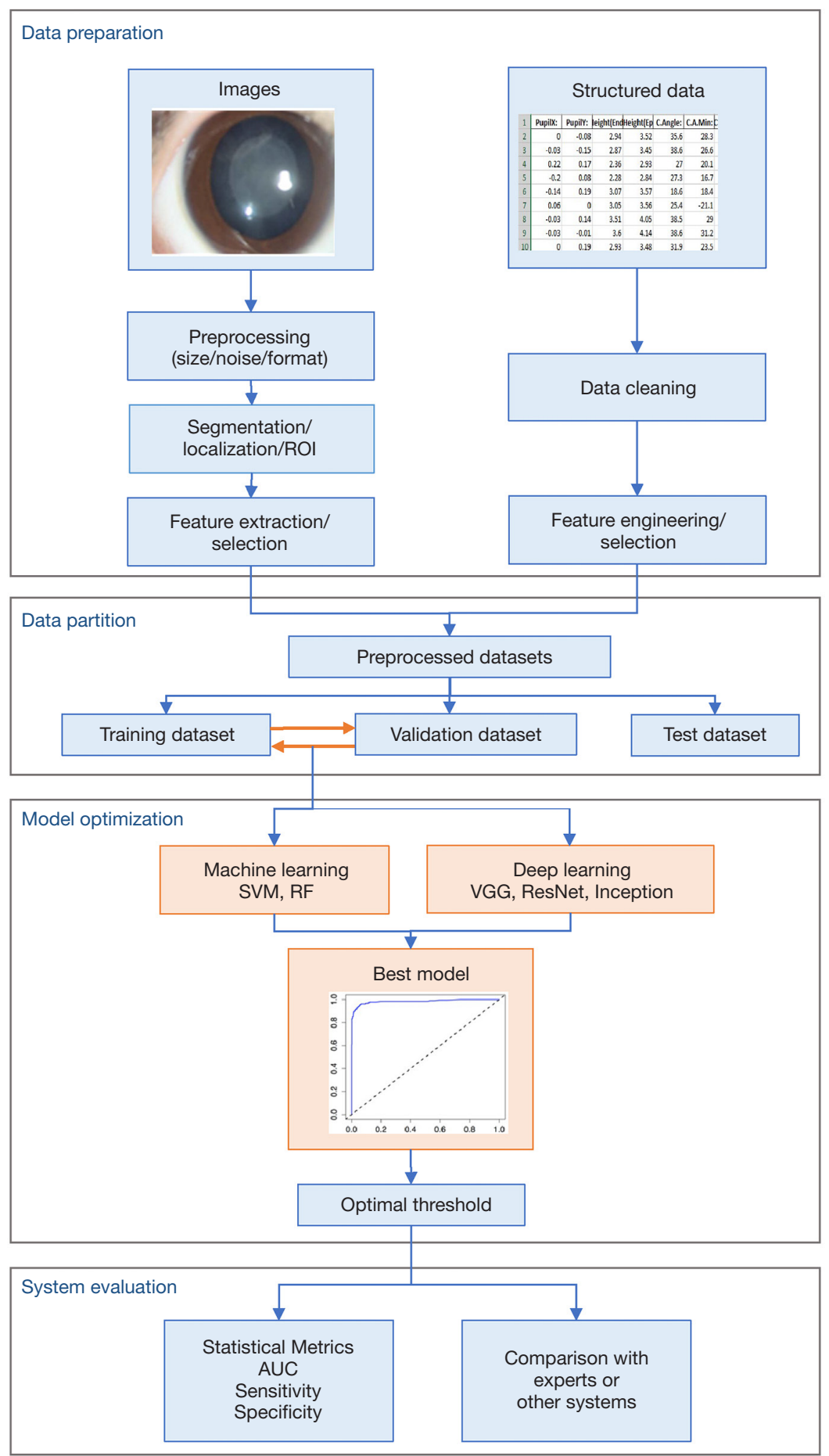

Figure 2 The workflow of constructing models. 
the condition of algorithms, it's crucial to prepare data well. Traditional ML algorithms only work on structured data, which require data preparation according to data types and $\mathrm{AI}$ algorithms. This is shown in Figure 2. The preprocessing and segmentation or location of region of interest (ROI) for images used in ML or DL for anterior segment ocular disease diagnosis have been the same. Image preprocessing involves reducing noise, for example specular reflection removal (34), eliminating uneven illumination (16), and transforming the images from different sources to same size and format, etc. Segmentation or location of ROI is essential for ML, but it is used only in some cases for DL because CNN has the ability to localize the discriminative regions used by it to identify the category (heatmap). Images for ML need to be converted to structured data, then the relevant features are usually selected and extracted to make learning more efficient and increase accuracy by avoiding overfitting. Structured data need data cleaning, feature engineering and selection. Data cleaning consists of outlier detection and removal, missing values imputation, examples out of target population removal, etc. Feature engineering is the process of using domain knowledge to create new features that make ML successful or outstanding. It is fundamental to the application of ML.

Data partition is the same for all data types and algorithms. Typically, data are randomly divided into two independent datasets: one is test dataset, the other will be divided again into training and validation dataset. This is called holdout method. Another method is cross-validation, of which 5-fold cross-validation has been commonly used in AI applications $(14,18,29,31,35,36)$ to avoid overfitting and underfitting when sample size is small. Training dataset is used to build models, validation dataset is for tuning the hyperparameters and selecting the optimal model as final model and making other decisions regarding the model, and test dataset is for evaluating the performance of the final model.

\section{Training and validating the model}

Training models are conducting experiments. Different classification algorithms $(13,15,16,18,23,24,27,28,31,35,37)$, hyperparameters, and data preparation methods have been adopted to train algorithms and generate several or even hundreds of models, then these models are evaluated on validation dataset so that the best model can be determined as the final model to use on future or unseen data. Other decisions, such as the optimal classification or operating threshold determined with the analysis of ROC curve (31), are also made based on the performance on validation dataset.

\section{Evaluating the model performance}

Model evaluation aims to estimate the performance of the final model on future data. The most commonly used metrics in AI for disease diagnosis are AUC [the area under the receiver's operator characteristics (ROC curve)], sensitivity, and specificity. AUC is more commonly used than accuracy because it isn't affected by the classification threshold or the proportion of positive examples as accuracy is. AUC ranges from 0.5 (for a model with no predictive value) to 1 (for a perfect model), and a value greater than 0.9 indicates the model is outstanding, 0.8-0.9 indicates the model is good or excellent (38). ROC curve is graphical display of sensitivity (true positive rate, TPR) on y-axis and (1 - specificity) (false positive rate, FPR) on $\mathrm{x}$-axis for varying cut-off points of probabilities predicted by the model. Except for examining the metrics on the testing dataset, comparing the performance of the model with human experts or other systems are also common, and ROC curve has been usually used to visually demonstrate the comparison of several test results simultaneously.

\section{Al algorithms}

Machine Learning is a paradigm that allows computers to learn patterns from a large corpus of data, and use the learned knowledge to make prediction. It can be roughly divided into traditional machine learning algorithms and deep learning algorithms. Traditional machine learning algorithms use variables selected by experts as input, and usually does not involve large neural networks. They include algorithms such as Linear Regression, Logistic Regression, Support Vector Machine, Decision Tree, Random Forest and etc. Deep learning algorithms make use of multimedia datasets, such as images, videos and sound, and usually involves the use of large-scale neural networks such as Artificial Neural Networks (ANNs), Convolutional Neural Networks (CNNs) and Recurrent Neural Networks (RNNs). While conventional machine learning methods are still employed, most recent researches focus on using deep neural networks.

Deep learning tasks mainly include three use cases: classification, object detection and semantic segmentation. In classification tasks, an algorithm learns to classify an image into a set of predefined categories. VGG, ResNet, 


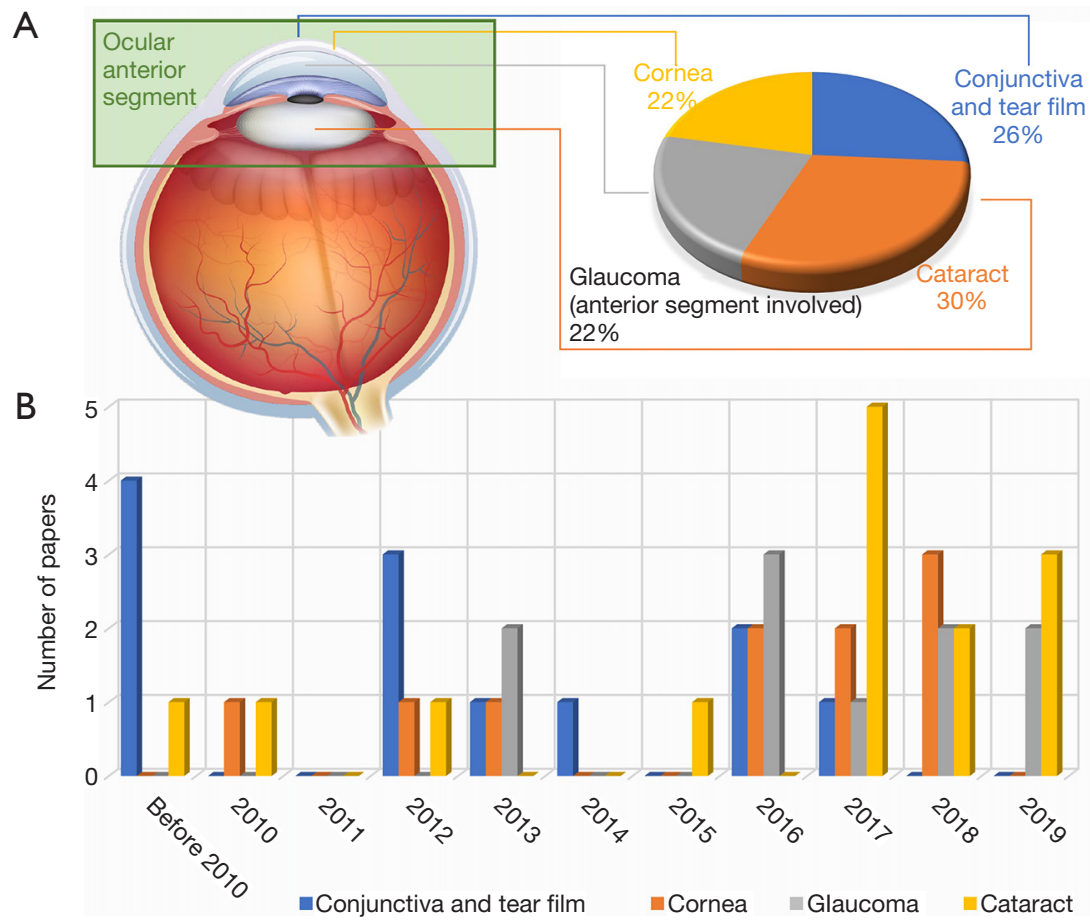

Figure 3 Publication of AI application in diagnosing anterior segment ophthalmic diseases. (A) Publication statistics per ophthalmological diseases. (B) Publication statistics per year (before Aug 1, 2019).

Inception and InceptionResNet are some of the popular CNNs used for classification.

The task of object detection focuses not only on what is in the image, but also on the position of the object(s) of interest. Popular object detection algorithms include FasterRCNN, You Only Look Once (YOLO) and Single Shot Multibox Detector (SSD).

In semantic segmentation, an algorithm tries to classify each pixel in an image into a set of predefined categories. Popular semantic segmentation algorithms include Fully Convolutional Network (FCN), U-Net and Google's DeepLab.

\section{Al application in anterior segment ophthalmic diseases}

A total of forty-five representative articles of AI application in anterior segment ophthalmic diseases are included in this review. Among them, the most intensively researched are conjunctiva and tear film, cornea, glaucoma and cataract. The distribution of the papers concerning these four topics in year of publication is shown in Figure 3. Details about aforementioned articles are summarized in http:// fp.amegroups.cn/cms/8e23864133337a312edb32e11e7655 dd/atm-20-976-1.pdf (39-53).

\section{Conjunctiva and tear film}

AI application in conjunctiva and tear film mainly focus on the diagnosis of dry eye. Dry eye is a multi-factorial disease characterized by unstable tear film causing a variety of symptoms and/or visual impairment, potentially accompanied by ocular surface damage (54). The diagnosis of dry eye is complicated and there is not a single perfect reference standard of it (55). Instead, a variety of examinations can provide supportive information, including fluorescein break up time test, tear film interferometry, tear film protein analysis and meibography.

Most investigations about dry eye were designed for the automatic analysis of relevant examinations $(24-27,40,42,56)$. Among these studies, more emphasis is on tear film interferometry. The interference patterns of tear film lipid layer can be analyzed by MLP, SVM and other algorithms to achieve automatic classification (24-27). Meibography, 
another imaging technology, has been investigated in another research (40). A combination of SIFT and SVM algorithms was applied to calculate the length and width of Meibomian glands in meibography images and then differentiate between healthy and unhealthy ones. Besides, fluorescein break up time test, a basic dry eye test, was also studied in $(42,56)$. By analyzing the recorded videos during fluorescein break up time test, break-up time (BUT) can be calculated (42) and dry areas detected (56) using MRF and RANSAC algorithms respectively.

Other than direct observation, $\mathrm{AI}$ algorithm was also applied to screen for the diagnostic candidate molecules. Grus et al. proved that analysis of tear film protein patterns by $\mathrm{ANN}$ can serve as a diagnostic tool for detection of dry eyes $(43,57)$. This artificial neural network showed an AUC of 0.93 and a specificity and sensitivity of approximately $90 \%$ for each (43), which provided a clue to accurate diagnosis of dry eyes.

Another research field lies in automatic conjunctival hyperemia grading, which is usually a subjective and timeconsuming procedure for humans. Using slit-lamp images or even ordinary eye images as input, ML methods are able to perform an objective assessment of hyperemia grading, removing both intra- and inter-expert subjectivity while providing a reduction in computation time $(37,39,41)$.

\section{Cornea}

Corneal ectasia, a group of eye disorders characterized by localized corneal thinning which leads to protrusion of the thinned cornea (58), seems to be the focus of AI research in cornea. Early detection of corneal ectasia is important since it is a contraindication for laser refractive surgery (59). A variety of AI models have been developed for the detection of corneal ectasia as a whole $(28,31)$ or one or more specific forms of it $(29,30,32,33,44)$, all of which were trained based on corneal parameters.

The most common form of corneal ectasia is keratoconus (60). Typically, keratoconus starts at puberty and may progress until the fourth decade or later (61). It's essential that keratoconus is detected at an early stage, so corneal collagen crosslinking can be applied in time, preventing a major deformation of cornea and potentially the need for a corneal transplant. Souza et al. found that SVM, MLP and RBFNN classifiers, when trained on Orbscan II data, could represent useful techniques for keratoconus detection from other non-keratoconus patterns with no differences between their performances (44).
Forme fruste keratoconus, or preclinical keratoconus, is an early and subclinical form of keratoconus. Although it is relatively stable with subtle topographic characteristics, it can decompensate after laser refractive surgery. Kovács et al. developed ML classifiers to discriminate eyes with preclinical keratoconus from normal eyes with an AUC of 0.96 (30), which is better than using unilateral single parameter.

More impressive works are from the multi-group classification of corneal conditions (29,32,33). Ruiz Hidalgo et al. developed a SVM algorithm to identify five different corneal patterns: keratoconus, forme fruste keratoconus, astigmatism, after refractive surgery and normal. For this 5 -group classification, the accuracy was $88.8 \%$, with a weighted average sensitivity of $89.0 \%$ and specificity of $95.2 \%$, suggesting the potential of this model to screen patients before refractive surgery.

Except for corneal ectasia, automatic diagnosis of corneal arcus is also studied. S et al. used a SVM algorithm to detect corneal arcus and cataracts, both of which are common among elderly people, in visible-wavelength images and obtained satisfactory results (34).

Few studies paid attention to keratitis and other inflammatory or degenerative corneal diseases on the basis of images classification. The interpretable and expandable deep learning system developed by Zhang et al. can identify keratitis and other ocular surface disorders, distinguish different anatomical parts and foci, discern the diagnostic information relevant to the diagnosis, and provide treatment suggestions (45).

\section{Glaucoma}

Glaucoma is a group of ocular disorders with multifactorial etiology united by characteristic intraocular pressure-associated optic neuropathy and is a leading cause of irreversible blindness worldwide $(62,63)$. Automatic detection of glaucoma-related features can contribute to timely diagnosis and thus improve patients' prognosis (62).

The two main types of glaucoma are open-angle glaucoma and angle-closure glaucoma. They can be differentiated by the structure of anterior chamber angle. Patients of both types usually experience elevations of intraocular pressure (IOP) and visual field defect throughout the disease. Aloudat et al. used decision tree and SVM to identify the presence of high IOP from frontal eye images (35). This framework achieved an overall accuracy of $95.5 \%$. As for analysis of visual defect, Wang et al. proposed 
an archetype method to detect visual field progression based on spatial pattern analysis and this method outperformed clinician-based evaluation in terms of accuracy and agreement (46). These studies provided helpful techniques for diagnosing glaucoma and monitoring its progression.

AS-OCT scans can detect the presence of angle closure and thus contribute to the diagnosis of angle-closure glaucoma. ML as well as DL algorithms have been applied for the detection of angle-closure in OCT images or parameters $(18-20,23,48)$. Fu et al. was the first to report the application of DL for this purpose (18). This DL system showed an AUC of 0.96 and proved a promising technology for the interpretation of OCT images. However, the usefulness of this system needs to be further validated in diverse population settings with the use of different devices. Additionally, two ML models trained on OCT images were also developed to classify the different mechanisms of angleclosure glaucoma $(21,22)$.

Compared with angle-closure glaucoma, the diagnosis of open-angle glaucoma is more difficult to be established since patients rarely experience symptoms until central visual field defect occurs. Previous studies found that certain parameters extracted from the 24-hour contact lens sensor(CLS) have been associated with the visual field progression rate recorded in treated glaucoma patients (64). Martin et al. deployed a RF algorithm to discriminate between primary open-angle glaucoma and healthy eyes using CLS parameters and IOP (47). The results demonstrated that the combined feature set achieved a significantly better performance than the IOP feature set. Accordingly, CLS parameters may contain information complementary to IOP for the differentiation of primary open-angle glaucoma from healthy eyes.

\section{Cataract}

A cataract is the loss of lens transparency due to opacification of the lens. Cataracts are the leading cause of visual impairment worldwide, accounting for more than $50 \%$ of blindness in lowincome and middle-income countries (65). Early detection and timely treatment of cataracts can improve patients' quality of life and reduce healthcare costs (66). The current standard of management of a visually significant cataract is surgical removal of the cataractous lens and its replacement with an intraocular lens (67). Among the complications of cataract surgery, posterior capsule opacification is the most common one, which can cause secondary visual loss (68).

Multiple ML and DL algorithms, including SVM,
DCNN and CRNN, have been applied for automatic diagnosis and grading of cataracts using different kinds of images such as slit-lamp images (10-15,36,45,49,50), visible wavelength images (34), and fundus images (16). The majority of aforementioned studies focused on a single specific cataract subtype, which can severely limit its application in real-world health-care settings. To address this problem, Wu et al. established a DL model to achieve the collaborative management of cataracts using a threestep strategy: capture mode recognition, cataract diagnosis and detection of referable cataracts with respect to etiology and severity (49). This AI agent showed satisfactory performance in the comprehensive diagnosis of agerelated cataracts, pediatric cataracts and posterior capsule opacification. Such a universal AI platform can be utilized in multilevel clinical scenarios and thus greatly facilitate the collaborative management of cataracts.

Apart from the usage of images, several ML prediction models using clinical and biometric variables have also been built (51-53). These models can be deployed to optimize the postoperative refractive outcome (53) and estimate the risk of pediatric cataracts (51) or posterior capsule opacification (52).

\section{Challenges and future prospects}

Despite the reported successes of AI application in anterior segment ocular diseases, several limitations and hurdles must be appropriately addressed before widespread clinical implementation. Although vast amounts of data are generated in daily ophthalmology care, they need to be properly prepared before being included in usable data sets. Trained professionals are required in this process to accomplish labeling, annotations, segmentations and quality assurance, making this process expensive in both time and cost. This issue is aggravated by data-hungry algorithms such as CNN. Thus, the development of algorithms that can learn with less data is in urgent need. Actually, many self-supervised, weakly-supervised and unsupervised methods have achieved satisfactory performance with a small dataset $(69,70)$, which can be a future trend in this field.

Besides the amount of data, the quality of data is also important and can greatly influence the performance of AI models $(71,72)$. Unfortunately, availability of high-quality data still presents a key challenge in the implementation of AI technologies in medicine $(73,74)$. Here, we propose a quality assessment system for images.

Before being included in data sets, images need to be scored in terms of the following items: being original or 
Table 1 Suggested assessment standards for images included in datasets

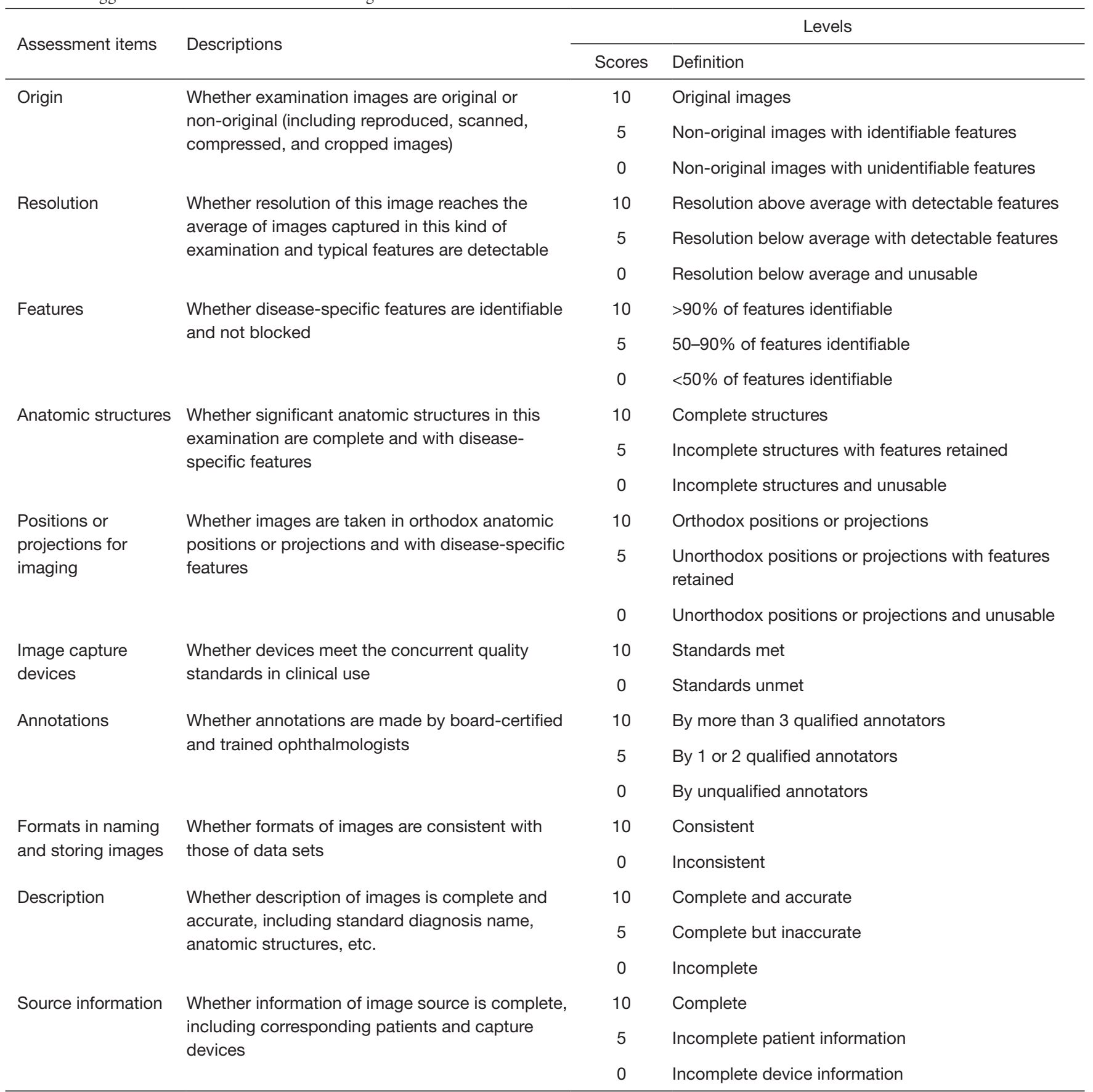

not, resolution, features, anatomic structures, positions or projections for imaging, image capture devices, annotations, formats in naming and storage, description, and source information. In each item, there are different levels of quality, with 10 referring the best and 0 referring the worst. Details of assessment items are shown in Table 1.
Furthermore, data sets in most AI studies are collected from a homogenous population, which can be a barrier to broader application. Ideally, AI models should be validated in larger data sets from various patient cohorts under different conditions. For this purpose, established data sets should be made more accessible to promote intellectual 
collaboration.

In addition to data issues, the characteristics of anterior segment ocular diseases also deserve more attention. For many anterior segment ocular diseases such as dry eye and glaucoma, it takes multiple examinations to add up to a definite diagnosis. However, current studies principally focus on automatic analysis of a single examination (like angle-closure detection in OCT images) rather than direct detection. For this reason, the integrative analysis of multimodal data (videos, images, formatting parameters, texts, etc.) to diagnose a complicated disease is an unsolved challenge in this field.

While it may take multiple examinations to diagnose one disease, different diseases can also be detected in a single examination and this is already partially realized in AI. For instance, a previous investigation showed that cataract and corneal arcus can both be detected in one slitlamp image by a SVM algorithm (34). The efficiency and applicability of such a multitasking AI system is much better than traditional binary classification models. What's more, slit-lamp images have also been used in other studies to accomplish conjunctiva hyperemia grading $(37,39,41)$ and high IOP detection (35), indicating the potential of this system to complete even more tasks. Accordingly, more efforts should be made to establish this kind of multitasking systems.

In contrast to the diagnosis of posterior segment ocular diseases, which usually requires ophthalmoscopy, detection of anterior segment ocular diseases is much more convenient. Several AI models already used ordinary eye images rather than slit-lamp images to achieve conjunctival hyperemia grading (41), detection of high IOP (35), corneal arcus and cataract (34). Based on these studies, it's possible that smartphone photography can serve as a diagnostic tool for anterior segment ocular diseases in the near future.

In conclusion, the applicability of AI in clinical settings can be improved by building systemic AI models trained on multimodal and heterogeneous data with a less datahungry algorithm. Furthermore, home-based diagnosis and management of diseases may also be realized someday. Although ethical, legal and regulatory issues remain to be settled, AI will undoubtedly play a crucial role in the revolution of current health care patterns.

\section{Acknowledgments}

Funding: This study was supported by the National Key Research and Development Program (2018YFC0116500), the Key Research Plan for the National Natural Science Foundation of China in Cultivation Project (91846109), the Science Foundation of China for Excellent Young Scientists (81822010), the National Natural Science Foundation of China (81770967, 81873675, 81800810), the Science and Technology Planning Projects of Guangdong Province (2019B030316012, 2018B010109008, 2017B030314025), Guangdong Science and Technology Innovation Leading Talents (2017TX04R031), the Natural Science Foundation of Guangdong Province (2018A030310104). The sponsor or funding organization had no role in the design or conduct of this research.

\section{Footnote}

Provenance and Peer Review: This article was commissioned by the Guest Editors (Haotian Lin and Limin Yu) for the series "Medical Artificial Intelligent Research" published in Annals of Translational Medicine. The article was sent for external peer review organized by the Guest Editors and the editorial office.

Conflicts of Interest: All authors have completed the ICMJE uniform disclosure form (available at http://dx.doi. org/10.21037/atm-20-976). The series "Medical Artificial Intelligent Research" was commissioned by the editorial office without any funding or sponsorship. HL served as the unpaid Guest Editor of the series. The other authors have no other conflicts of interest to declare.

Ethical Statement: The authors are accountable for all aspects of the work in ensuring that questions related to the accuracy or integrity of any part of the work are appropriately investigated and resolved.

Open Access Statement: This is an Open Access article distributed in accordance with the Creative Commons Attribution-NonCommercial-NoDerivs 4.0 International License (CC BY-NC-ND 4.0), which permits the noncommercial replication and distribution of the article with the strict proviso that no changes or edits are made and the original work is properly cited (including links to both the formal publication through the relevant DOI and the license). See: https://creativecommons.org/licenses/by-nc-nd/4.0/.

\section{References}

1. Graham N. Artificial intelligence. Blue Ridge Summit, Pa.: 
Tab Books, 1979.

2. Samuel AL. Some Studies in Machine Learning Using the Game of Checkers. IBM J Res Dev 1959;3:20.

3. LeCun Y, Bengio Y, Hinton G. Deep learning. Nature 2015;521:436-44.

4. Abràmoff MD, Lou Y, Erginay A, et al. Improved Automated Detection of Diabetic Retinopathy on a Publicly Available Dataset Through Integration of Deep Learning. Invest Ophthalmol Vis Sci 2016;57:5200-6.

5. Gulshan V, Peng L, Coram M, et al. Development and Validation of a Deep Learning Algorithm for Detection of Diabetic Retinopathy in Retinal Fundus Photographs. JAMA 2016;316:2402-10.

6. Poplin R, Varadarajan AV, Blumer K, et al. Prediction of cardiovascular risk factors from retinal fundus photographs via deep learning. Nat Biomed Eng 2018;2:158-64.

7. Lakhani P, Sundaram B. Deep Learning at Chest Radiography: Automated Classification of Pulmonary Tuberculosis by Using Convolutional Neural Networks. Radiology 2017;284:574-82.

8. Esteva A, Kuprel B, Novoa RA, et al. Dermatologist-level classification of skin cancer with deep neural networks. Nature 2017;542:115-8.

9. Ting DSW, Peng L, Varadarajan AV, et al. Deep learning in ophthalmology: The technical and clinical considerations. Prog Retin Eye Res 2019;72:100759.

10. Acharya RU, Yu W, Zhu K, et al. Identification of cataract and post-cataract surgery optical images using artificial intelligence techniques. J Med Syst 2010;34:619-28.

11. Gao X, Lin S, Wong TY. Automatic Feature Learning to Grade Nuclear Cataracts Based on Deep Learning. IEEE Trans Biomed Eng 2015;62:2693-701.

12. Lin H, Li R, Liu Z, et al. Diagnostic Efficacy and Therapeutic Decision-making Capacity of an Artificial Intelligence Platform for Childhood Cataracts in Eye Clinics: A Multicentre Randomized Controlled Trial. EClinicalMedicine 2019;9:52-9.

13. Liu X, Jiang J, Zhang K, et al. Localization and diagnosis framework for pediatric cataracts based on slit-lamp images using deep features of a convolutional neural network. PLoS One 2017;12:e0168606.

14. Long E, Lin H, Liu Z, et al. An artificial intelligence platform for the multihospital collaborative management of congenital cataracts. Nat Biomed Eng 2017;1:0024.

15. Wang L, Zhang K, Liu X, et al. Comparative analysis of image classification methods for automatic diagnosis of ophthalmic images. Sci Rep 2017;7:41545.

16. Xu X, Zhang L, Li J, et al. A hybrid global-local representation $\mathrm{CNN}$ model for automatic cataract grading. IEEE J Biomed Health Inform 2020;24:556-67.

17. Zhang K, Liu X, Liu F, et al. An Interpretable and Expandable Deep Learning Diagnostic System for Multiple Ocular Diseases: Qualitative Study. J Med Internet Res 2018;20:e11144.

18. Fu H, Baskaran M, Xu Y, et al. A Deep Learning System for Automated Angle-Closure Detection in Anterior Segment Optical Coherence Tomography Images. Am J Ophthalmol 2019;203:37-45.

19. Fu H, Xu Y, Lin S, et al. Segmentation and Quantification for Angle-Closure Glaucoma Assessment in Anterior Segment OCT. IEEE Trans Med Imaging 2017;36:1930-8.

20. Huazhu F, Yanwu X, Wong DW, et al. Automatic anterior chamber angle structure segmentation in AS-OCT image based on label transfer. Conf Proc IEEE Eng Med Biol Soc 2016;2016:1288-91.

21. Niwas SI, Lin W, Bai X, et al. Automated anterior segment OCT image analysis for Angle Closure Glaucoma mechanisms classification. Comput Methods Programs Biomed 2016;130:65-75.

22. Niwas SI, Lin W, Kwoh CK, et al. Cross-Examination for Angle-Closure Glaucoma Feature Detection. IEEE J Biomed Health Inform 2016;20:343-54.

23. Nongpiur ME, Haaland BA, Friedman DS, et al. Classification algorithms based on anterior segment optical coherence tomography measurements for detection of angle closure. Ophthalmology 2013;120:48-54.

24. Peteiro-Barral D, Remeseiro B, Mendez R, et al. Evaluation of an automatic dry eye test using MCDM methods and rank correlation. Med Biol Eng Comput 2017;55:527-36.

25. Remeseiro B, Bolon-Canedo V, Peteiro-Barral D, et al. A methodology for improving tear film lipid layer classification. IEEE J Biomed Health Inform 2014;18:1485-93.

26. Remeseiro B, Penas M, Barreira N, et al. Automatic classification of the interferential tear film lipid layer using colour texture analysis. Comput Methods Programs Biomed 2013;111:93-103.

27. Remeseiro B, Penas M, Mosquera A, et al. Statistical comparison of classifiers applied to the interferential tear film lipid layer automatic classification. Comput Math Methods Med 2012;2012:207315.

28. Ambrósio R Jr, Caiado AL, Guerra FP, et al. Novel pachymetric parameters based on corneal tomography for diagnosing keratoconus. J Refract Surg 2011;27:753-8. 
29. Arbelaez MC, Versaci F, Vestri G, et al. Use of a Support Vector Machine for Keratoconus and Subclinical Keratoconus Detection by Topographic and Tomographic Data. Ophthalmology 2012;119:2231-8.

30. Kovács I, Mihaltz K, Kranitz K, et al. Accuracy of machine learning classifiers using bilateral data from a Scheimpflug camera for identifying eyes with preclinical signs of keratoconus. J Cataract Refract Surg 2016;42:275-83.

31. Lopes BT, Ramos IC, Salomao MQ, et al. Enhanced Tomographic Assessment to Detect Corneal Ectasia Based on Artificial Intelligence. Am J Ophthalmol 2018;195:223-32.

32. Ruiz Hidalgo I, Rodriguez P, Rozema JJ, et al. Evaluation of a Machine-Learning Classifier for Keratoconus Detection Based on Scheimpflug Tomography. Cornea 2016;35:827-32.

33. Smadja D, Touboul D, Cohen A, et al. Detection of Subclinical Keratoconus Using an Automated Decision Tree Classification. Am J Ophthalmol 2013;156:237-46.e1.

34. S V MK, R G. Computer-Aided Diagnosis of Anterior Segment Eye Abnormalities using Visible Wavelength Image Analysis Based Machine Learning. J Med Syst 2018;42:128.

35. Aloudat M, Faezipour M, El-Sayed A. High Intraocular Pressure Detection from Frontal Eye Images: A Machine Learning Based Approach. Conf Proc IEEE Eng Med Biol Soc 2018;2018:5406-9.

36. Jiang J, Liu X, Zhang K, et al. Automatic diagnosis of imbalanced ophthalmic images using a cost-sensitive deep convolutional neural network. Biomed Eng Online 2017;16:132.

37. Sánchez Brea ML, Barreira Rodriguez N, Sanchez Marono $\mathrm{N}$, et al. On the development of conjunctival hyperemia computer-assisted diagnosis tools: Influence of feature selection and class imbalance in automatic gradings. Artif Intell Med 2016;71:30-42.

38. Lantz B. Machine Learning with R. UK: Packt Publishing Ltd, 2013.

39. Sánchez Brea ML, Barreira Rodriguez N, Mosquera Gonzalez A, et al. Defining the Optimal Region of Interest for Hyperemia Grading in the Bulbar Conjunctiva. Comput Math Methods Med 2016;2016:3695014.

40. Koh YW, Celik T, Lee HK, et al. Detection of meibomian glands and classification of meibography images. J Biomed Opt 2012;17:086008.

41. Derakhshani R, Saripalle SK, Doynov P. Computational methods for objective assessment of conjunctival vascularity. Conf Proc IEEE Eng Med Biol Soc
2012;2012:1490-3.

42. Yedidya T, Carr P, Hartley R, et al. Enforcing monotonic temporal evolution in dry eye images. Med Image Comput Comput Assist Interv 2009;12:976-84.

43. Grus FH, Podust VN, Bruns K, et al. SELDI-TOF-MS ProteinChip array profiling of tears from patients with dry eye. Invest Ophthalmol Vis Sci 2005;46:863-76.

44. Souza MB, Medeiros FW, Souza DB, et al. Evaluation of machine learning classifiers in keratoconus detection from orbscan II examinations. Clinics (Sao Paulo) 2010;65:1223-8.

45. Wu XL, Qiu QC, Liu Z, et al. Hyphae Detection in Fungal Keratitis Images With Adaptive Robust Binary Pattern. IEEE Access 2018;6:13449-60.

46. Wang M, Shen LQ, Pasquale LR, et al. An Artificial Intelligence Approach to Detect Visual Field Progression in Glaucoma Based on Spatial Pattern Analysis. Invest Ophthalmol Vis Sci 2019;60:365-75.

47. Martin KR, Mansouri K, Weinreb RN, et al. Use of Machine Learning on Contact Lens Sensor-Derived Parameters for the Diagnosis of Primary Open-angle Glaucoma. Am J Ophthalmol 2018;194:46-53.

48. Xu Y, Liu J, Cheng J, et al. Automated anterior chamber angle localization and glaucoma type classification in OCT images. Conf Proc IEEE Eng Med Biol Soc 2013;2013:7380-3.

49. Wu X, Huang Y, Liu Z, et al. Universal artificial intelligence platform for collaborative management of cataracts. Br J Ophthalmol 2019;103:1553-60.

50. Jiang J, Liu X, Liu L, et al. Predicting the progression of ophthalmic disease based on slit-lamp images using a deep temporal sequence network. PLoS One 2018;13:e0201142.

51. Long E, Xu S, Liu Z, et al. Construction and implications of structural equation modeling network for pediatric cataract: a data mining research of rare diseases. BMC Ophthalmol 2017;17:74.

52. Mohammadi SF, Sabbaghi M, Z-Mehrjardi H, et al. Using artificial intelligence to predict the risk for posterior capsule opacification after phacoemulsification. J Cataract Refract Surg 2012;38:403-8.

53. Findl O, Struhal W, Dorffner G, et al. Analysis of nonlinear systems to estimate intraocular lens position after cataract surgery. J Cataract Refract Surg 2004;30:863-6.

54. Tsubota K, Yokoi N, Shimazaki J, et al. New Perspectives on Dry Eye Definition and Diagnosis: A Consensus Report by the Asia Dry Eye Society. Ocul Surf 2017;15:65-76.

55. Savini G, Prabhawasat P, Kojima T, et al. The challenge of 
dry eye diagnosis. Clin Ophthalmol 2008;2:31-55.

56. Yedidya T, Hartley R, Guillon JP, et al. Automatic dry eye detection. Med Image Comput Comput Assist Interv 2007;10:792-9.

57. Grus FH, Augustin AJ. Analysis of tear protein patterns by a neural network as a diagnostical tool for the detection of dry eyes. Electrophoresis 1999;20:875-80.

58. Romero-Jiménez M, Santodomingo-Rubido J, Wolffsohn JS. Keratoconus: a review. Cont Lens Anterior Eye 2010;33:157-66; quiz 205.

59. Bower KS, Woreta F. Update on contraindications for laser-assisted in situ keratomileusis and photorefractive keratectomy. Curr Opin Ophthalmol 2014;25:251-7.

60. Sachdev G, Sachdev MS, Sachdev R, et al. Unilateral corneal ectasia following small-incision lenticule extraction. J Cataract Refract Surg 2015;41:2014-8.

61. Rabinowitz YS. Keratoconus. Surv Ophthalmol 1998;42:297-319.

62. Weinreb RN, Aung T, Medeiros FA. The pathophysiology and treatment of glaucoma: a review. JAMA 2014;311:1901-11.

63. Casson RJ, Chidlow G, Wood JP, et al. Definition of glaucoma: clinical and experimental concepts. Clin Exp Ophthalmol 2012;40:341-9.

64. De Moraes CG, Jasien JV, Simon-Zoula S, et al. Visual Field Change and 24-Hour IOP-Related Profile with a Contact Lens Sensor in Treated Glaucoma Patients. Ophthalmology 2016;123:744-53.

65. Lee CM, Afshari NA. The global state of cataract blindness. Curr Opin Ophthalmol 2017;28:98-103.

66. Limwattananon C, Limwattananon S, Tungthong J, et al.

Cite this article as: $\mathrm{Wu} \mathrm{X}$, Liu L, Zhao L, Guo C, Li R, Wang T, Yang X, Xie P, Liu Y, Lin H. Application of artificial intelligence in anterior segment ophthalmic diseases: diversity and standardization. Ann Transl Med 2020;8(11):714. doi: 10.21037/atm-20-976
Association Between a Centrally Reimbursed Fee Schedule Policy and Access to Cataract Surgery in the Universal Coverage Scheme in Thailand. JAMA Ophthalmol 2018;136:796-802.

67. Liu YC, Wilkins M, Kim T, et al. Cataracts. Lancet 2017;390:600-12.

68. Thompson AM, Sachdev N, Wong T, et al. The Auckland Cataract Study: 2 year postoperative assessment of aspects of clinical, visual, corneal topographic and satisfaction outcomes. Br J Ophthalmol 2004;88:1042-8.

69. Zhai X, Oliver A, Kolesnikov A, et al. S4L: Self-Supervised Semi-Supervised Learning. arXiv e-prints 2019. Available online: https://arxiv.org/abs/1905.03670

70. Vo HV, Bach F, Cho M, et al., editors. Unsupervised image matching and object discovery as optimization. Proceedings of the IEEE Conference on Computer Vision and Pattern Recognition; 2019.

71. Lee JG, Jun S, Cho YW, et al. Deep Learning in Medical Imaging: General Overview. Korean J Radiol 2017;18:570-84.

72. Ting DSW, Cheung CY, Lim G, et al. Development and Validation of a Deep Learning System for Diabetic Retinopathy and Related Eye Diseases Using Retinal Images From Multiethnic Populations With Diabetes. JAMA 2017;318:2211-23.

73. He J, Baxter SL, Xu J, et al. The practical implementation of artificial intelligence technologies in medicine. Nat Med 2019;25:30-6.

74. Rajkomar A, Dean J, Kohane I. Machine Learning in Medicine. N Engl J Med 2019;380:1347-58. 